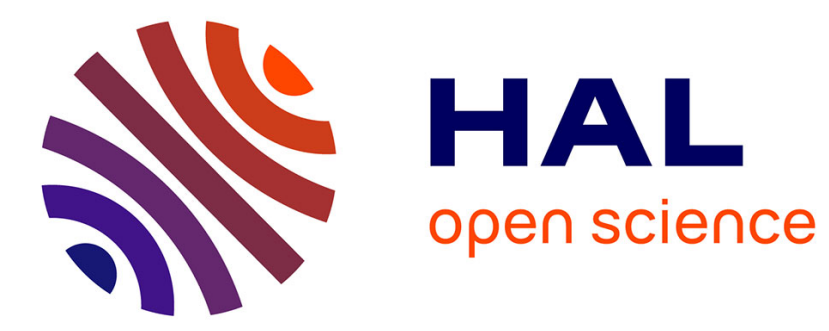

\title{
L'éthique du care malmenée au cours des interactions entre infirmiers et individus sans-abri \\ Laureline Coulomb
}

\section{To cite this version:}

Laureline Coulomb. L'éthique du care malmenée au cours des interactions entre infirmiers et individus sans-abri . Revue des Sciences sociales, 2014, Vers une société du CARE?, 52, pp.86-91. hal-01295087

\section{HAL Id: hal-01295087 \\ https://hal.science/hal-01295087}

Submitted on 30 Mar 2016

HAL is a multi-disciplinary open access archive for the deposit and dissemination of scientific research documents, whether they are published or not. The documents may come from teaching and research institutions in France or abroad, or from public or private research centers.
L'archive ouverte pluridisciplinaire HAL, est destinée au dépôt et à la diffusion de documents scientifiques de niveau recherche, publiés ou non, émanant des établissements d'enseignement et de recherche français ou étrangers, des laboratoires publics ou privés. 
REVUE DES

SGIENGES

SOMMAIRE

VERS UNE SOCIÉTÉ DU CARE? 2014 n52

PRÉSENTATION

p.4 BERNARD WOEHL, PHILPPE HAMMAN \& FREDDY RAPHAËL

p. 8 JUAN MATAS

p.80 CAROLINE GIACOMONI, HÉLEENE HOARAU

.12 DANIEL BERTAUX \& CATHERINE DEL CROIX

DES FLUX MIGRATOIRES DE TRAVAILLEUSES DU CARE

p. 18 HELMA WTZ \& EWA PALENGA-MÓLLENBECK divisée. Liens et transnationa

JUAN MATAS

(n)

D. 42 NATACHA BORGEAUD-GARCIANDA Relations de care, affects ef dominations. Le care à

FRANCE -ALLEMAGNE

ÉTUDES COMPARATIVES DE FORMES DE CARE

D.54 JANINA GLAESER

Assistmilesen methe et politiques de la petite entance : une comparaiso France-Allemagne

p.62 JULLE SENTIS

Apprendre les métiers du care en France et en Allemagne

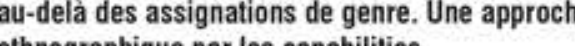

LE CARE EN INSTITUTION

TROIS ÉTUDES DE CAS EN FRANCE

p.72 MOUNRFARHAT

MOUNIR FARHAT personnel et distance professionnelle en unité Alzheimer

P. 80 CAROLINE GIACOMONI, HÉLEENE HOARAU
\& ALAIN MONNEREAU Enjeux et conflits autour du care à travers l'expérience de la délégation à domicile des traitents en concérologie

p. 86 LAURELINE COULOMB Léthique du care malmenée au cours des interactions entre infirmiers et individus sans-abri LUTTES ET RÉFORMES POTENTIELLES

p. 94 URSULA APITZSCH net l'ordre de genre

p. 102 AGNËS BERTHELOT-RAFFARD La discrimination par association : une expression du care dominé

p. 110 CHANTAL NICOLE-DRANCOUR Pour une reconnaissance du care dans des sociétés de pleine activité

p. 118 DANEL BERTAUXX

政 CHANTIERS DE RECHERCHE

p. 132 ÉLOOIE VALENTIN

Emotions, dynamiques citoyennes et espace public. L'expérience du projet social d'une maison de quartier a Dunkerque

p. 142 SUZY GUTH Conflit dans le vignoble : Ammerschwihr et l'AOC Kaefferkopf

LU - À LIRE

p. 154 Recensions

p. 166 Résumés des articles

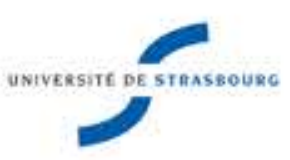

Cirs

Revve bénéficiant du soutien de l'Isstitut
des Sciences Humaines el Sociales du CNRS
SSN 1623-6572

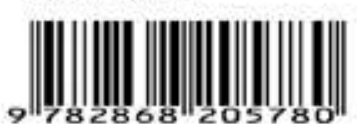

REVUE DES SOIENOES SOBALES

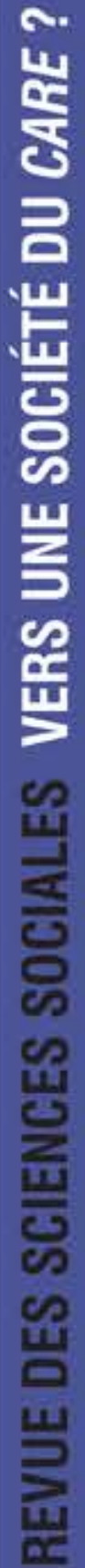

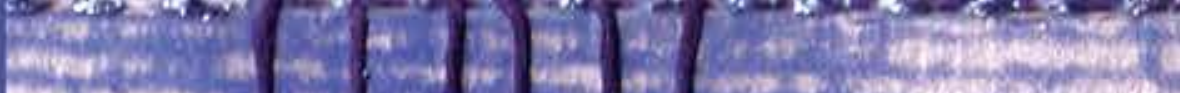

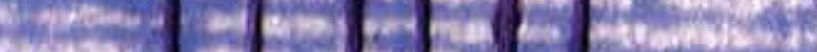

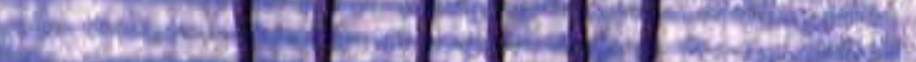
6.

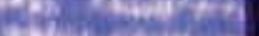
wast:

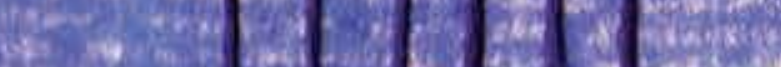

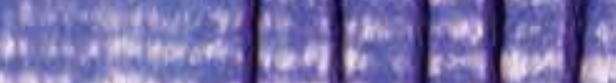

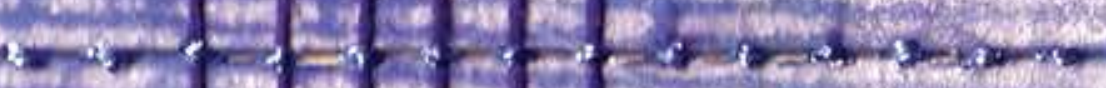
-

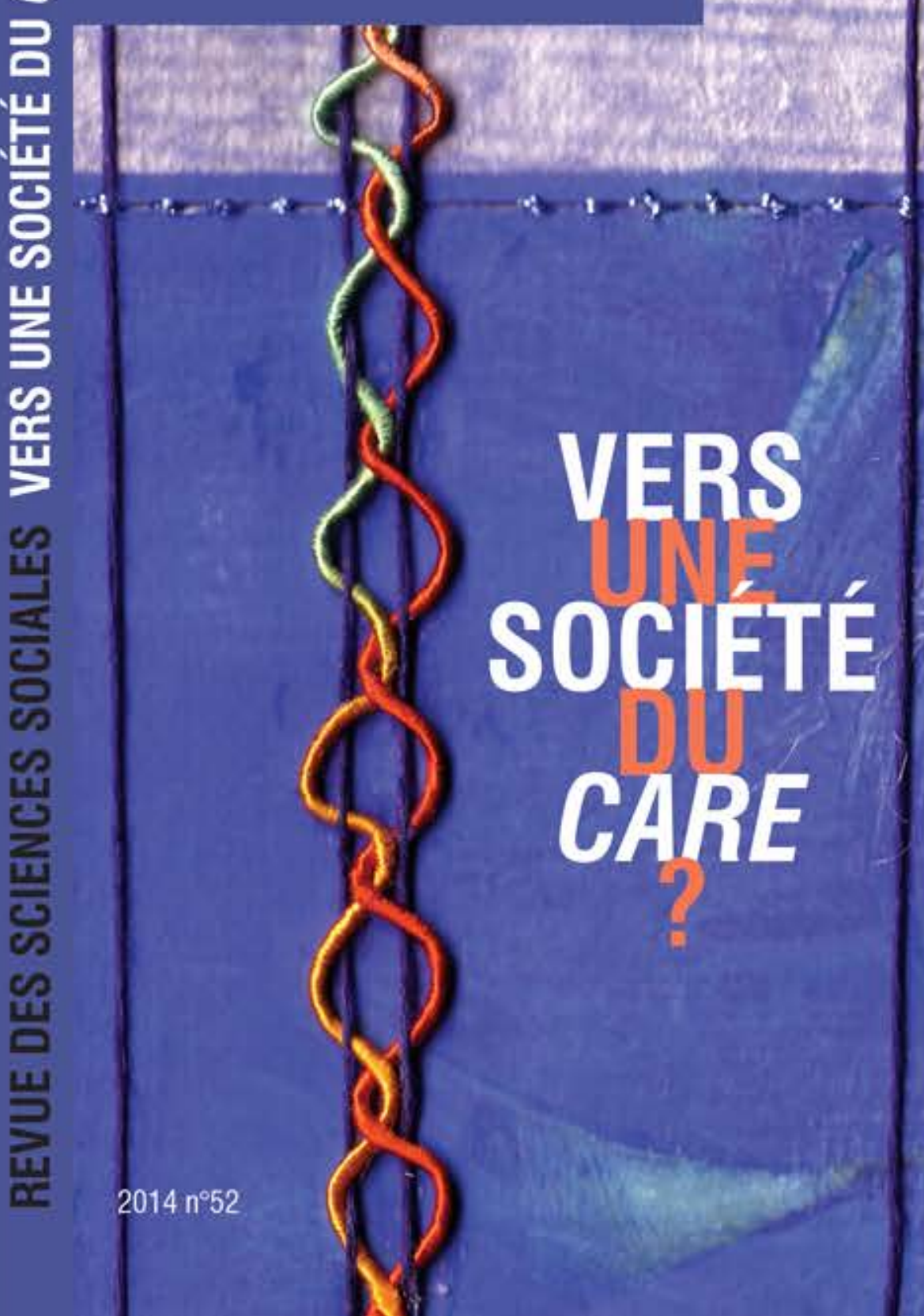


REVUE DES

SAIN

SOGALES

VERS UNE SOCIÉTÉ DU CARE ?

$2014 n^{\circ 5}$ ?

- Directeur scientifique

Freddy Raphaël

- Rédacteur en che

Patrick Ténoudji

- Comité scientifique

Gerges Balandier (EHESS Paris)

(CENRS Toulouse).

(MaymTP Paris) Gionas Paris), Jean Cuisenier

Milano). Jose Carios Gomes da Silva (Portugal).

François Hèran (INED Paris), Claude Javeau (Univ. Libre

de Bruxelles). Nicole Lapierre (EHESS/CNRS Paris).

Marianne Mesnil (Univ. Libre de Bruxelles).

Sonia Montecino (Univ. de Chile), Jean Rèmy (Univ.

Alain Tarrius (Univ. Toulouse-Le Mirail), Alain Touraine

(CEMS Paris)

- Comité de rédaction

Chelle Biannuis-Gasser (Univ. Tours), Maurice Blane Univ. Strasbourg), Nicoletta Diasio (Univ. Strasbourg). Woltong Strasbourg). Antida Gazzola (Univ. Genova). Philippe Hamman (Univ. Strasbourg). Pascal Hintermeyer (Univ. Strasbourg), Leila Jeolas (Uniy Londrina), Reinhard Johler (Univ, Túbingen). Salvatore La Mendola (Univ. Padova), David Le Breton (Univ. Strasbourg/UF), Juan Malas (Univ. Strasbourg. Gabriele Profita (Univ. Palerme), Ilario Rossi (Univ. Lausanne), Patrick Schmoll (CNRS Strasbourg), Roger Some (Uni. Slasbourg), Simona tersign

Collaborateurs éditoriaux

Anny Bloch, Marie-Noële Dents,

Anny Bloch, Marie--Noële Der
- Équipe iconographique sur ce numéro

Vincent Hans, Aline Mathy, Patrick Schmo

- Ce numero a été coordonné par :

Catherine Delcroix, Juan Matas \& Daniel Bertaux

- Maquette

Couverture : Aline Mathy

Presses Universitimires de Stroshour

- Administration

Presses Universitaires de Strasboutro

5 allée du Gal Rouvillois - CS 50008

FR-67083 Strasbourg Cedex

Tél. 0368856265

e-mail : pu-strasbourg@unistra.tr

- Diftusion/distribution

agences d'abonnement :

cid@msh-paris.tr

(nstitutions:

www.lcdpu.fi

18 rue Robert-Schuman

CS 90003

FR-94227 Charenton-le-Pont Ceder

Tel. 0153485630

Revue publiée par la Faculté des sciences sociale

de TUniversité de Strasbourg et le laboratioire

DST 1023.0572 (2)

www.revue-des-sciences-sociales.com
Ál'attention des auteurs

Les articles sont à adresser par document attachẻ à l'attention de Patrick Ténouditi, rédacteur en chêt <patrick.tenoudji@misha.fr>

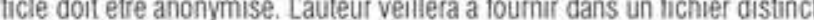

- sestitres ou protession et son appantenance instiutionnelle (université, centre de recherche)

- un résumé d'une dizaine de lignes en français et en anglais.

Le texte comporte au maximum 40000 signes et blancs, notes et bibliographie comprises. II est Myhme par des intertitres courts, avec deux niveaux dintertitres au maximum. Les passages cites de rextes en langues tlangertes sont traduns en lançais. Les nolts, oni

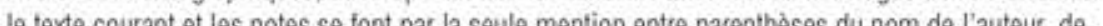
l'année de publication et, le cas échéant, des numéros de pages citées.

Exemples

- ... a ainsi que lindique J. Dupont (2003a) ...

-.... cette question a lairt objel de plusieurs travaux (Oupont 2003a, Durand 2004. 2007) ...

La bibliographie suit les consignes de présentations suivantes :

- pour un ouvage . Oupont J. (2003a), Thre de rouvrage en iltafique, Lieu d'édition, Editeur.

- pour un chapitse douvrage : Durand M. (2004). Titre du chapitre sans guillemets, in Dupont

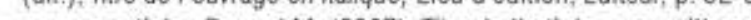

-

Les articles soumis par les auteurs sont examinés chacun par un comité d'au moins deux lecteurs qui rendent des avis séparés. Lexemplaire du texte qui leur est remis ne mentionne pas lidentité de Tauteur, et lauteur de son cote ne peut se laire communiquer T identitie de ses lecteurs : la decision

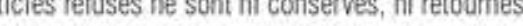

Si des graphiques et illustrations sont nécessaires à l'appui du texte, l'auteur en adresse les originaux ou les fichiers image haute résolution (300dpi) à la rédaction. Hors ce cas de figure, la recherche la rédaction de la revue. 


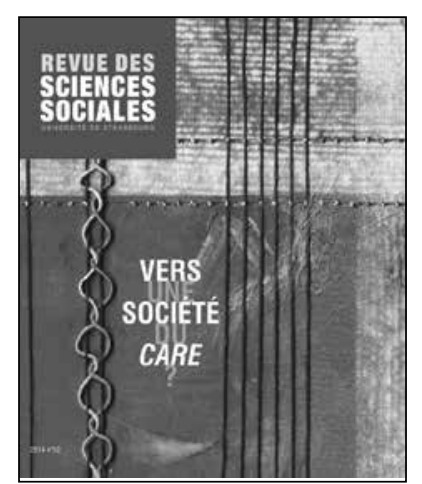

Khadija Seddiki

artiste plasticienne, diplômée de l'Académie royale des beaux-arts de Belgique, de l'Académie des arts et métiers

Constantin Meunier d'Etterbeek, des Écoles supérieures des beaux-arts d'Alger et d'Oran, et de l'Université Denis Diderot-Paris VII. Elle a fondé TISSART, école d'art et atelier de tissage, à Vanves. http://khadijaseddiki.com/
Dans les sociétés du "Nord" de la planète, les besoins de care augmentent alors que les femmes n'acceptent plus guère de s'y consacrer sans contrepartie. Ce déséquilibre engendre des flux importants et différenciés de migrantes venant de «l'Est» ou du «Sud». Qui sont-elles, dans quelles conditions viennent-elles, dans quels cadres, lieux, rapports et conditions concrètes travaillent-elles, quels sont leurs droits?

Ce numéro examine la situation de travailleuses du care: Polonaises en Allemagne, Péruviennes à Buenos Aires ou au Chili; il aborde les contextes institutionnels de ces activités en France, en Allemagne, dans une perspective comparative. Une "société du care» est-elle en train d'émerger?
Couverture

image: Khadija Seddiki composition: Aline Mathy 


\section{PRÉSENTATION}

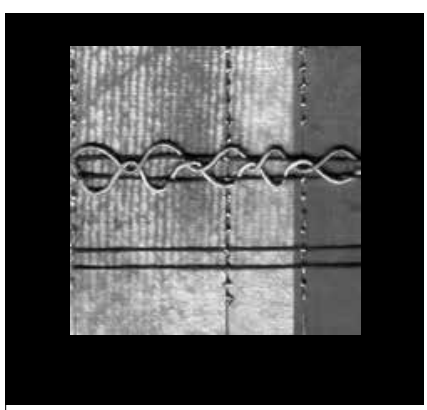

4

BERNARD WOEHL

PHILIPPE HAMMAN \& FREDDY RAPHAËL

Hommage

à François Steudler 8

JUAN MATAS

Introduction

12

DANIEL BERTAUX \& CATHERINE DELCROIX

Présentation
DES FLUX MIGRATOIRES DE TRAVAILLEUSES DU CARE

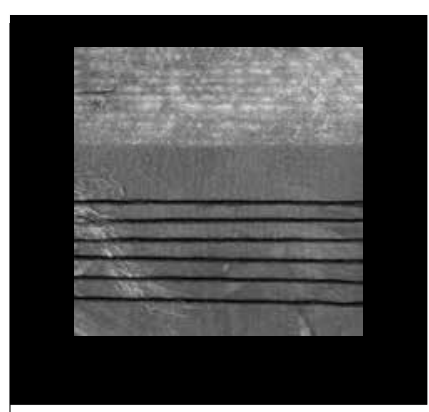

18

HELMA LUTZ \&

EWA PALENGA-

MÖLLENBECK

Les migrantes du care dans

l'Europe divisée. Liens et

contradictions dans un

espace transnational

\section{8}

\section{JUAN MATAS}

Les migrantes péruviennes au Chili et le travail de care

opportunités et risques

d'une situation en plein essor

\section{2}

NATACHA BORGEAUD-

\section{GARCIANDA}

Relations de care, affects et dominations. Le care à demeure à Buenos Aires
FRANGE -ALLEMAGNE : ÉTUDES COMPARATIVES DE FORMES DE CARE

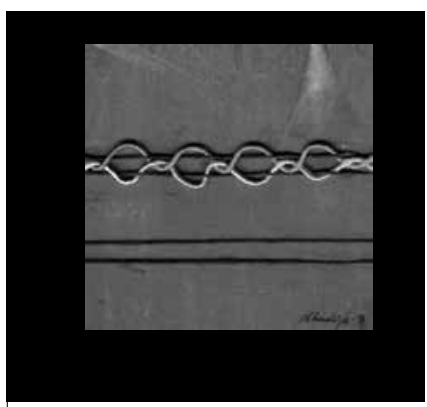

54

\section{JANINA GLAESER}

Assistant(e)s maternel(le)s d'origine étrangère et politiques de la petite enfance: une comparaison France-Allemagne

\section{2}

\section{JULIE SENTIS}

Apprendre les métiers du care en France et en Allemagne au-delà des assignations de genre. Une approche ethnographique par les capabilities
LE GARE EN INSTITUTION : TROIS ÉTUDES DE CAS EN FRANGE

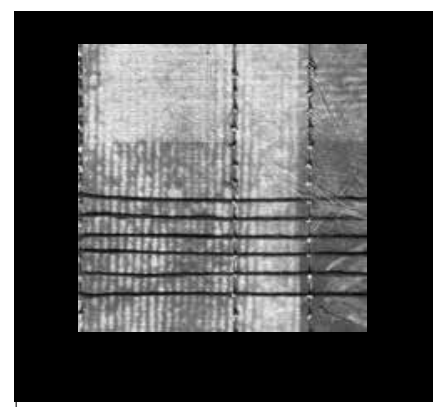

72

\section{MOUNIR FARHAT}

Un dilemme du care:

tension entre engagement personnel et distance professionnelle en unité Alzheimer

80

CAROLINE GIACOMONI, HÉLĖNE HOARAU \& ALAIN MONNEREAU

Enjeux et conflits

autour du care à travers

l'expérience de la délégation à domicile des traitements en cancérologie

86

\section{LAURELINE COULOMB}

L'éthique du care malmenée au cours des interactions entre infirmiers et individus sans-abri 


\section{LUTTES ET RÉFORMES POTENTIELLES}

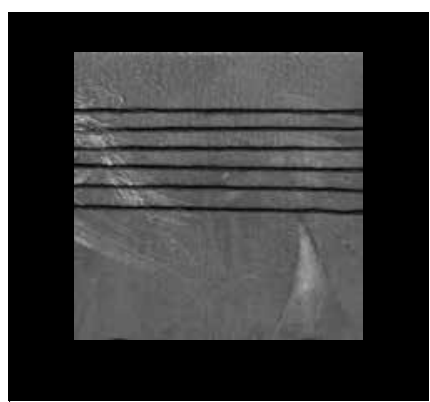

94

URSULA APITZSCH

Le care, la migration

et l'ordre de genre

102

AGNÈS BERTHELOT-RAFFARD

La discrimination

par association:

une expression

du care dominé

110

CHANTAL NICOLE-

\section{DRANCOURT}

Pour une reconnaissance

du care dans des sociétés

de pleine activité

\section{8}

\section{DANIEL BERTAUX}

Le care comme partie

émergée de la production

de la vie

\section{CHANTIERS} DE RECHERCHE

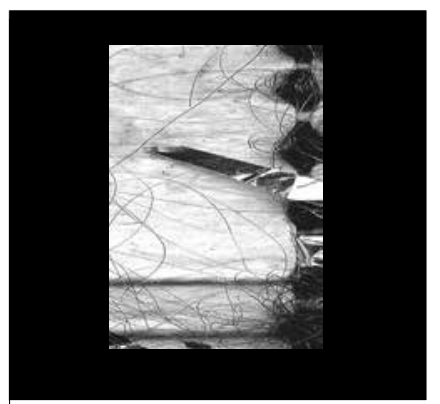

132

ELODIE VALENTIN

Émotions, dynamiques

citoyennes et espace public. L'expérience du projet social

d'une maison de quartier à

Dunkerque

\section{2}

\section{SUZY GUTH}

Conflit dans le vignoble:

Ammerschwihr

et I'AOC Kaefferkopf
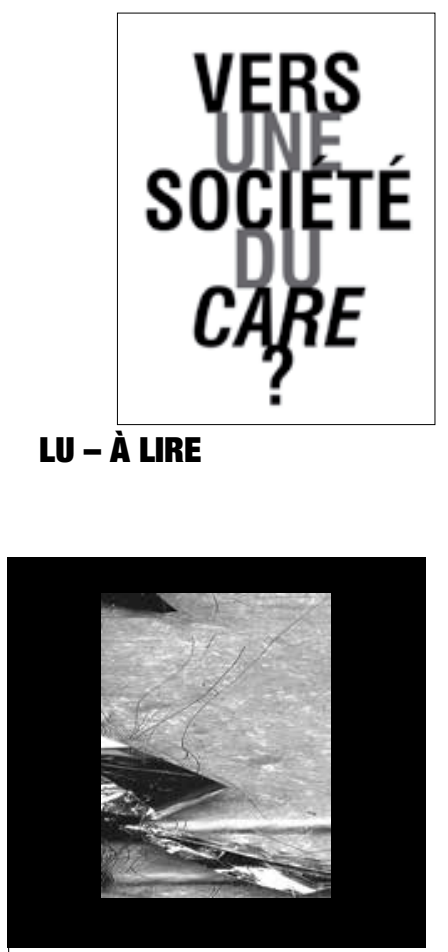

154

RECENSIONS

166

RÉSUMÉS DES ARTICLES 


\section{L'éthique} du care malmenée

\section{au cours des interactions entre infirmiers et individus sans-abri}

e care permet de regrouper sous un seul dénominatif un

ensemble de dispositions, d'activités, de conceptions éthiques qui ont toutes en commun d'être tournées vers l'autre. Il implique de reconnaître la vulnérabilité ontologique de l'individu, "car c'est dans la vulnérabilité que réside ce qui motive le Moi à témoigner du care pour autrui, puisqu'il est conscient de sa dépendance constitutive» (Pulcini 2012, p. 56). Pour réussir à élaborer une définition du care qui soit le plus fidèle possible à la réalité sociale, Joan Tronto avance que «la meilleure manière de penser le care est sans doute de l'envisager comme pratique. La notion de pratique est complexe; c'est une alternative aux conceptions du care comme principe ou émotion. Désigner le care comme une pratique implique qu'il est à la fois pensée et action, que l'une et l'autre sont étroitement liées et orientées vers une certaine fin» (Tronto 2008, p. 251). Les pratiques de care englobent donc l'éthique portée par les dispensateurs du care, les valeurs qu'ils défendent ainsi que leurs activités résultant de l'application de leurs principes moraux. Dans le cadre des activités de soin, le care correspond à la prise en charge du patient dans sa globalité et son individualité: son corps et sa pathologie, mais aussi sa personnalité, sa souffrance, son passé et son futur.

Les relations entre infirmiers et personnes sans-abri se montrent parfois compliquées tant leurs modes de raisonnement sont éloignés. En effet, leurs valeurs et représentations du monde social divergent, ce qui crée de nombreux malentendus qui peuvent s'avérer conflictogènes. Au cours de leurs interactions, les valeurs du care sont malmenées, voire reléguées au second plan. Ces situations peuvent entraîner des comportements infirmiers qui entrent en contradiction avec leur éthique du care, tandis que les soignés se sentent infantilisés, délaissés, voire maltraités. Leurs vulnérabilités respectives sont dès lors exacerbées et leurs relations sont encore fragilisées. Pourtant, la relation soignant-soigné est déterminante pour la prise en charge médicale des patients dans des conditions optimales, aussi bien pour le personnel de santé que pour les patients.

Cet article se base sur des observations menées dans différentes structures de soin strasbourgeoises, spécialisées ou non dans la prise en charge d'individus sans-domicile. Ces observations sont complétées par des entretiens semi-directifs menés parallèlement auprès de soignants et de personnes sans-abri. Nous essaierons d'adopter la même posture que $S$. Fainzang dans son étude portant sur l'information et le mensonge dans la relation médecin-patient: "le souci est de tenter de comprendre ce que médecins d'un côté et malades de l'autre disent pour justifier leurs actes et d'analyser leurs conduites sans les juger, dans une relation d"'empathie ethnologique" à l'égard des deux parties, sans choisir d'endosser le discours de l'une plutôt que celui de l'autre, tous deux considérés comme valides, avec leurs raisons et leurs logiques propres» (Fainzang 2006, p.9). Ce positionnement empathique est heuristique car il permet au chercheur de se placer dans une optique compréhensive des situations. De plus, il contribue à la neutralité axiologique du chercheur, qui va appréhender les expériences des acteurs en partant de leur point de vue, autant que possible, et limite ainsi le sentiment de jugement chez les interviewés et observés. 
L'éthique du care des infirmiers est malmenée au cours des interactions entre soignants et soignés sans-abri. En premier lieu, la demande de soin des individus sans-domicile est difficilement interprétable, notamment parce qu'ils semblent rechercher autant de reconnaissance que de soins médicaux. Ensuite, la fragilité identitaire des soignants peut les mettre en contradiction avec leurs valeurs du care lorsqu'ils sont en difficulté, voire en incapacité de prendre en charge leurs patients de manière optimale.

\section{La demande de soin sibylline des personnes sans-domicile}

Les parcours de vie des individus sans-abri, souvent très mouvementés et modelés par de nombreuses ruptures, peuvent les conduire à développer "une manière d'être spécifique» (Le Breton 1992). Leur rapport au corps est malmené à la rue et ils souffrent de leur manque de reconnaissance. En effet, il est difficile pour les individus en très grande précarité de garder une identité sociale avantageuse. Les principaux dénominatifs utilisés pour les définir caractérisent le manque: sans-emploi, sans-domicile, sans-papier, sans-abri, sanslogement... et leurs possibilités de se valoriser, d'obtenir une certaine reconnaissance sociale sont réduites. Ils peuvent alors adopter des stratégies de valorisation de soi basées sur leur corps et sa mise en scène. Ils l'utilisent pour démontrer leur résistance physique et insistent souvent sur le caractère choisi de leur vie à la rue. Ils utilisent, dans leur discours, une rhétorique particulière dont le but est de montrer à l'interlocuteur qu'ils sont capables d'endurer bien davantage que les autres: les conditions de vie à la rue. Ils racontent alors les nuits d'hiver, la manche sous la pluie, la faim, la violence de la rue, leur résistance à la douleur... comme autant de preuves de leur robustesse. Les personnes sans-domicile se conforment plus que quiconque aux injonctions de paraître fort et résistant, ce type de comportement représente une forme de défense par rapport aux difficultés de la vie à la rue. Elena Pulcini rappelle à ce sujet que «la parabole de l'individualisme moderne et du sujet souverain a fini par masquer, ou plutôt par aboutir au refoulement de cette condition ontologique de vulnérabilité qui, une fois admise, peut pousser le sujet à reconnaître son insuffisance et sa dépendance par rapport à autrui, le fait qu'il est inéluctablement lié aux autres, à d'autres vies, à d'autres destins." (Pulcini 2012, p. 54-55). Les comportements et discours des individus sansdomicile s'orientent autour de ce que Pascale Pichon appelle la «carrière de survie» (Pichon 2010) et répondent à une même problématique: «comment tenir lorsque tout s'écroule autour de soi? Comment justifier ce que l'on est devenu? Comment maintenir une image de soi respectable?" (Pichon 2010 , p. 52). Les savoirs et savoir-faire spécifiques de la vie à la rue (où trouver à manger, comment installer l'endroit où l'on dort pour moins souffrir du froid, comment protéger ses affaires du vol...) servent à mettre en scène la résistance physique et psychologique qui permet de survivre à la rue. Ils fournissent un dernier rempart contre le sentiment d'échec qui caractérise la vie dans la rue et la minimisation des souffrances, la dissimulation des fragilités individuelles sont une des pierres fondatrices de ce rempart.

Cette rhétorique de la robustesse se retrouve de manière globale dans leurs discours et également lors des interactions avec des soignants. Ils peuvent alors préférer camoufler leurs plaies, étouffer leurs symptômes, pour ne pas paraître faibles. Pourtant, l'état de santé des individus sans-domicile est, de manière générale, très altéré et ils sont nombreux à avoir besoin de soin. Or, pour demander de l'aide et accepter qu'un autre prenne soin de soi, il est nécessaire d'accepter d'être en situation de demande par rapport à l'autre. "Une partie des raisons pour lesquelles nous préférons méconnaître les formes routinières du care en tant que tel est de préserver l'image que nous avons de nous-mêmes comme n'étant pas soumis au besoin. L'état de besoin étant conçu comme une menace pour notre autonomie, ceux qui ont davantage de besoins que nous-mêmes nous paraissent moins autonomes et donc comme disposant de moins de pouvoirs et de capacités. Il en résulte qu'une des manières dont nous interprétons socialement la situation de ceux qui ont besoin du care est de les considérer comme pitoyables, parce qu'ils demandent de l'aide" (Tronto 2008, p. 257). Les individus sans-abri cherchent à éviter ce type de relation pour ne pas être considérés comme inférieurs. ils peuvent renoncer aux soins, refuser qu'un soignant prenne soin d'eux afin d'éviter d'être définis une fois de plus par la négative, comme quelqu'un qui ne peut se prendre en charge lui-même. La compréhension de leur demande de soin devient alors délicate pour les soignants, en effet, cette ambivalence de comportement pousse les soignants à devoir déchiffrer l'attente des individus sans-domicile. Leur besoin de soin est exprimé de manière sibylline, ils cherchent à se faire soigner tout en essayant de prouver qu'ils n'en ont pas besoin. Pour ne pas perdre la face, ils minimisent leurs douleurs et leurs symptômes en utilisant la rhétorique du «gros dur». Les infirmiers risquent alors de ne pas comprendre leur demande de soin, ou de ne prendre en charge que les symptômes que les patients dévoilent, sans savoir qu'ils en dissimulent d'autres.

Une distinction entre le cure et le care s'opère plus facilement en anglais qu'en français. Ces deux termes permettent de différencier ce qui est de l'ordre du prendre soin, de manière globale (le care) et du soin spécifiquement médical (le cure). Il ressort des observations de terrain et des entretiens que les individus en très grande précarité attendent en priorité, de la part des soignants, du care, c'est-àdire que l'on prenne soin d'eux, avant même de traiter leurs pathologies. En effet, il semble qu'ils recherchent davantage à être reconnus comme individu plutôt que d'être soignés. En prenant le rôle de malade, ils peuvent laisser de côté celui de SDF. Leur nouveau rôle leur permet une certaine reconnaissance: ils sont désormais 
traités comme des personnes dont il faut prendre soin, dans leur individualité et leur particularité. Ils quittent momentanément l'invisibilité sociale dont ils souffrent à la rue. À l'hôpital, ils ont de la valeur puisqu'on cherche à les soigner. Monsieur A., par exemple, insiste sur l'importance de traiter les patients comme des hommes avant tout. À la question "qu'attendez-vous d'un infirmier?», il répond:

"Il doit être fidèle à sa profession, traiter le malade comme un être humain et pas comme un objet. [...] Le malade est homme et pas un objet, pas un clochard, pas un rien, un fainéant... il est homme. » (Monsieur A., Roumain, environ 60 ans, rencontré en centre d'accueil de jour).

Il recherche une relation d'égal à égal, dans laquelle il n'est pas étiqueté comme «clochard». Monsieur E., quant à lui, insiste sur la dimension relationnelle du soin. Dans son discours, la relation apparaît comme un préalable au soin et lorsque celui-ci est prodigué sans aucune dimension relationnelle, comme, dans son exemple, les piqûres distribuées à la chaîne, cela pose problème:

"Y'a [des infirmiers] qui sont plus doux, ils ont plus le sens des relations, ils arrivent à mettre les gens à l'aise, et après ça se passe bien! Y'en a qui... juste à la tâche, tac tac tac! Hop, la piqûre et je men vais! C'est sûr que souvent ça passe pas avec les gens." (Monsieur E., Marocain, environ 50 ans, rencontré en centre d'accueil de jour).

Il faut d'abord «que ça passe avec les gens" pour que le soin puisse s'opérer. La composante relationnelle du soin apparaît prégnante dans ce qu'attendent les personnes sans-abri des personnels médicaux et soignants, comme si la relation avec le soignant apaisait leurs maux aussi bien que les soins médicaux. On retrouve chez les infirmiers cette même importance accordée à la relation inter-individuelle et au temps nécessaire à la mettre en place:

«Dans le milieu hospitalier, pour moi le côté relationnel était essentiel! Pour d'autres, absolument pas! Je trouve que les infirmières qui nont pas entre guillemets ce "sens relationnel" ne sont pas de bonnes infirmières, même si elles sont peut-être de bonnes techniciennes... Parce que ce côté relationnel c'est super important, même pour nous qui sommes voilà... Qui pouvons entre guillemets "nous défendre”, c'est important l'écoute... Donc le bon soignant, à l'hôpital, peut aussi bien être un bon technicien mais avoir... Ce côté relationnel euh... pouvoir prendre le temps, l'écoute... » (Madame G., infirmière en centre d'accueil de jour).

Lors des interactions de soins avec des patients sdf, la relation soignantsoigné prend une importance accrue. Ces patients particuliers peuvent mettre en difficulté les infirmiers tant leurs comportements sont pétris d'ambivalence: ils oscillent entre la nécessité d'obtenir des soins, leur état de santé étant généralement très dégradé et la volonté de paraître forts et résistants.

Les attentes de soin des personnes de la rue correspondent non seulement aux soins médicaux, mais aussi, voire surtout, à la prise en charge de leur besoin de reconnaissance. On observe une forme de paralipse dans le discours des personnes sans-domicile, la rhétorique qu'ils emploient semble à la fois crier leur souffrance tout en cherchant à la camoufler à tout prix. Par exemple, Monsieur R. (environ 40 ans, rencontré en centre d'hébergement médicalisé) explique que la douleur n'est pas un problème pour lui et, pour le prouver, il donne un grand coup sur son épaule blessée et se contente de grimacer pour réagir à la douleur. Pourtant son épaule est véritablement abîmée et il cherche à la faire soigner. Ce type de comportement peut avoir une incidence sur le diagnostic et le traitement qui vont lui être proposés, car sa demande de soin devient très compliquée à interpréter du fait de ce double discours: il demande des soins pour sa douleur tout en essayant de démontrer qu'elle ne l'atteint pas. C'est grâce à l'établissement d'une relation de confiance que l'individu sans-domicile va accepter de se dévoiler et de mettre à nu ses souffrances.

\section{La fragilité identitaire des infirmiers}

Les conditions d'exercice professionnel difficiles des soignants les mettent parfois en contradiction avec leurs valeurs de care. En effet, ils apportent une importance capitale à la relation établie avec le patient et expliquent que, pour être un bon infirmier, il est nécessaire de conjuguer des qualités tant techniques que relationnelles. Or, les injonctions de rapidité et d'efficacité qui leur sont faites peuvent les forcer à minimiser la qualité de cette relation. Les individus sans-abri pâtissent de ce manque de relation, bien qu'ils comprennent les difficultés des soignants. Monsieur H., estime que les soignants n'ont pas les moyens de consacrer du temps à leurs malades:

"Une bonne infirmière, c'est celle qui n'a pas trop de pression pour pouvoir soccuper correctement des patients quelle a! à ce qui paraît c'est pas trop le cas! Enfin on ne leur en donne pas trop l'occasion... » (Monsieur H., Français, 28 ans, rencontré en centre d'accueil de jour).

De plus, les patients rencontrent des difficultés de compréhension du diagnostic ou des soins. Elles s'expliquent par leur imparfaite maîtrise de la langue, mais également par des logiques d'appréhension particulière de la maladie. En effet, il est fréquent que les individus sans-abri ne conçoivent la réalité de leur pathologie qu'à partir du moment où elle entrave leur quotidien, c'est-à-dire lorsqu'ils ne leur est plus possible de réaliser leurs activités habituelles à cause de symptômes devenus handicapants. Les soignants ont alors une charge de travail supplémentaire: leur fonction d'explication des soins, de la pathologie devient bien plus conséquente. Cette dimension particulière du soin, qui consiste à faire comprendre au patient le diagnostic et l'intérêt des soins, revêt une importance capitale car elle constitue un préalable à l'adhésion aux soins des patients. Par exemple, Monsieur R. explique avoir été hospitalisé plusieurs mois, mais ne sait pas de quoi il souffrait: 
"C'est comme si quand quelqu'un tombe dans le coma, il sait pas où il est, il voit rien. C'est comme s'il voit blanc ou noir ou jaune. Et jai rien compris. Je me suis réveillé au bout de trois mois, je savais même pas ce qui mest arrivé. C'est ça que jai pas compris! [...] Un jour jen avais ras le cul de la perfusion, jarrache la perfusion, y'a le sang qui a giclé dans toute la chambre! Apres y'a linfirmière qui est venue, oh! Elle a gueulé avec moi! J'ai dit "vas te faire foutre! J'en ai rien à foutre moi!" " (Monsieur R., 58 ans, Français, rencontré en structure d'hébergement médicalisé)

$\mathrm{Ne}$ sachant pas de quoi il souffre ni à quoi servent les traitements, il les a refusé en allant jusqu'à arracher sa perfusion. Il explique plus loin dans l'entretien qu'on l'a transféré dans un autre service. Il a alors adhérer aux soins grâce aux infirmières qui s'occupaient de lui:

«Là-bas je me suis fait chouchouter par les jeunes infirmières! Ahlala! Alors là ceétait quelque chose de classe! Même des très jolies! Je me suis pas... douté de ça! Mais que je me suis fait chouchouter par eux! Mais au moins, eux se sont occupé de moi!»

Il insiste sur le fait que les infirmières de ce service, se sont occupées de lui: il a donc le sentiment qu'on ne se préoccupait pas de lui auparavant. Les patients sans-domicile et les infirmiers souffrent pareillement de ce manque de temps et de relation. Ces derniers se retrouvent dans un contexte de tension lorsque les soignés sans-abri n'expriment pas de demande de soin claire, voire refusent les soins lorsqu'ils n'en comprennent pas l'intérêt. Les soignants ont alors l'impression d'imposer des soins aux patients, ce qui entre en contradiction avec leur éthique du care. Cette situation les amène à une importante fragilité identitaire, qui s'explique à la fois par leurs conditions de travail pénibles, leur sentiment de manque de reconnaissance et leurs difficultés à faire concorder leur travail effectif avec leur éthique du care. Lors des entretiens, nombreux sont ceux à définir leurs conditions de travail comme difficiles, tant au niveau physique que psychologique. Par exemple, Madame
C., cadre de santé dans un service de réanimation médicale, raconte:

"Tu sais yavait des mois où on avait une dizaine de morts: on pouvait avoir trois, quatre, cing morts dans la semaine! [...] Ce qui fait que t'as souvent une vision tres pessimiste des choses. [...] Et physiquement... C'est du sport! Parce que l'infirmière qui arrive le matin, quelques fois, jusqu’à $14 \mathrm{~h}$, elle n'a pas arrêté une minute. Elle est dans le stress constant! Et puis quelques fois avec une impression de ne pas maîtriser les choses. Parce que la vie téchappe, et quoi que tu fasses, la vie téchappe." (Madame C., cadre de santé, 58 ans).

Elle décrit précisément l'épuisement physique et psychologique dont peuvent souffrir les soignants. Ils sont confrontés à une charge de travail très importante, qui ne leur laisse pas de répit. À la fatigue physique s'ajoute la difficulté à supporter psychologiquement les situations auxquelles ils doivent faire face. Un sentiment d'inutilité est évoqué: quel que soit l'investissement des infirmiers, il leur est souvent impossible de remédier à la souffrance de leurs patients, ou d'empêcher leur décès.

\section{Rasséréner le care}

Dans ce contexte, les patients aux prises en charge complexes représentent une difficulté supplémentaire pour les soignants. Anne Véga explique alors, dans ses recherches sur les représentations des infirmières hospitalières, qu'ils risquent d'étiqueter ces patients sous le terme de "mauvais" malades». Il s'agit prioritairement des patients aux prises en charge longues, avec des pathologies multiples et nécessitant beaucoup de soins. L'étiquette du " "mauvais" malade» apparaît lorsque les soignants sont dans l'incapacité d'apporter une réponse de soin aux patients. "Les "mauvais" malades sont, du point de vue infirmier, des personnes qui demandent une prise en charge trop importante, vis-à-vis desquels l'infirmière ne peut ni investir son rôle curatif, ni développer son rôle "maternel", relationnel. Ainsi, les mauvais malades sont sou- vent qualifiés de malades "lourds": on retrouve, parmi eux, les toxicomanes, grabataires, SDF, malades du sida, les personnes venant des urgences ou de psychiatrie, en stade terminal de cancer..., autant de catégories dépréciées dans la société, souvent incurables et/ ou demandant de nombreux soins de nursing, voire des prises en charge sociales» (Véga 1997, p. 115-116). Lors des entretiens, la catégorisation entre «bons" et "mauvais» patients est globalement rejetée par les infirmiers interrogés, cependant, elle est utilisée pour définir le patient qui les met en difficulté. La volonté de ne pas juger les malades selon qu'ils soient «bons» ou «mauvais» résulte des valeurs et de l'éthique du care des infirmiers. Mais en situation de stress professionnel, cet étiquetage fait surface et reflète les sentiments d'incapacité et d'inutilité, dont ils souffrent sur le terrain, et qui les mettent en contradiction avec leur propre éthique du care. Lorsqu'ils ne voient pas d'amélioration de l'état de santé du patient, que leurs soins de care leur semblent inefficaces, ils risquent de reléguer leur rôle relationnel au second plan, car, isolé du cure, celui-ci leur paraît souvent futile. Les infirmiers sont démunis face aux patients dont les problématiques sociales et de reconnaissance sont aussi importantes que celles de l'ordre du médical. En effet, ils n'ont pas la possibilité d'assurer ce type de prise en charge: ils n'en ont ni les moyens pratiques, ni la formation requise; et malgré la présence d'assistants de service social dans chaque service hospitalier, les solutions proposées restent souvent insuffisantes, notamment lorsqu'il s'agit d'individus sans-domicile pour lesquels les possibilités d'hébergement et de logement à la sortie de l'hospitalisation sont plus que limitées. Le manque de solutions d'hébergement pour ces patients accentue le sentiment d'inutilité des infirmiers. Ils dispensent des soins en sachant qu'au sortir de l'hospitalisation, leur patient retournera vivre dans la rue où son état de santé risque fort de se dégrader presque aussitôt.

Lorsque soignants et soignés sansdomicile sont en co-présence, pour reprendre les termes de Giddens, il 
arrive que leurs relations soient tendues. Les interactions de soin comportent en effet une dimension conflictogène tant les fragilités des uns et des autres ressurgissent. L'entrechoquement de leurs fragilités respectives entraîne souvent un comportement défensif de la part des infirmiers et des personnes de la rue, qui tous deux risquent de voir leur sécurité ontologiquel menacée. Ce qui se joue à vrai dire c'est qu'ils ne partagent pas leurs logiques d'action et éprouvent des difficultés à comprendre les comportements de l'autre: ils peuvent interpréter de manière erronée certaines attitudes, en les prenant par exemple comme des marques de non-respect. Ce phénomène les met en difficulté car, comme nous le rappelle Anthony Giddens, «les agents assurent et maintiennent la prépondérance du tact, de la confiance ou de la sécurité ontologique en déployant un ensemble ahurissant d'habiletés dans la production et la reproduction de l'interaction. De telles habiletés reposent d'abord et avant tout sur le contrôle, régularisé par des normes, de ce qui peut sembler n'être, encore plus qu'un ordre de succession, que les plus minuscules et les plus insignifiants détails d'expression et de mouvements du corps» (Giddens 2012 , p. 128). Or, dans notre cas, les deux parties en présence ne partagent pas les mêmes normes, nécessaires à la préservation de leur sécurité ontologique, et ils peuvent alors interpréter ces minuscules et insignifiants détails d'expression et de mouvements du corps comme des signes d'indifférence ou d'irrespect de la part de l'autre. Par exemple, M.J. déplore être mal accueilli aux urgences:

"Les infirmières, elles me connaissent bien et quand jarrive, elles me disent directement "pshht! Dans le bloc 16!", mais moi jai dit "hé! On dit pas pshht! On dit d'abord bonjour!"”. (Monsieur J., environ 40 ans, rencontré en maraude).

Monsieur souffre d'une épilepsie importante ainsi que d'une forte dépendance à l'alcool et cette situation entraîne des consultations récurrentes auprès des services hospitaliers d'urgences. Mais bien qu'il se rende fréquemment dans ces services et qu'il est probable que les infirmières le reconnaissent, on imagine difficilement qu'il serait envoyé directement dans un bloc sans autre forme de diagnostic. Cependant, son propos illustre bien qu'il se sent dévalorisé au cours de ses interactions avec les infirmières et qu'il attache une importance particulière à la relation d'égal à égal avec le soignant. L'échange doit s'entamer par un «bonjour» réciproque qui est déjà une forme de care. Si l'on se place du point de vue du soignant, il est probable que celui-ci ne se sente pas reconnu selon la qualité et la pénibilité de son travail. Ainsi, soignant et soignés sont mis à mal. Ils n'ont pas les mêmes attentes l'un envers l'autre et leurs logiques de fonctionnement sont très éloignées, leur compréhension mutuelle est donc altérée et l'éthique du care est malmenée car les infirmiers ne peuvent faire concorder leurs actions effectives à leurs valeurs du soin.

Pourtant des dispositifs particuliers permettent d'atténuer ces difficultés, bien que sans toujours réussir à y pallier entièrement. Les Lits Halte Soins Santé par exemple offrent un espacetemps particulier qui ne souffre pas des mêmes contraintes organisationnelles qu'un service hospitalier. Les personnes de la rue y sont hébergées et accompagnées dans leurs démarches de soin et d'accès au droit pour une durée de prise en charge de deux mois renouvelables. Ainsi, les soignants vont se concentrer davantage sur le care, perçu comme un préalable au cure. Une adaptation progressive à la structure peut être observée dans le comportement des résidents qui vont peu à peu accepter de révéler leurs symptômes et leurs difficultés. Les comportements des soignants évoluent également au cours de la prise en charge: alors qu'ils se concentrent initialement sur le care du résident pour répondre à son besoin de reconnaissance et le faire rester dans la structure, les soignants vont s'intéresser davantage au cure en entamant une démarche d'éducation aux soins, une fois qu'une relation de confiance a pu s'instaurer. Cet espace-temps permet la création d'une relation soignant-soigné préservant autant que possible la sécurité ontologique de chacun, grâce aux marges de liberté accordées aux uns comme aux autres. Les demandes de soin des individus sans-domicile peuvent s'exprimer et les soignants ont la possibilité de prendre le temps de les comprendre et d'y répondre. Ce type de dispositif représente alors un espace-temps intermédiaire favorable au soin et au rassérènement du care.

Les interactions entre infirmiers et individus sans-domicile révèlent souvent les fragilités identitaires de chacun. Au contact l'une de l'autre, elles s'exacerbent et compromettent leurs relations. Les conditions effectives de prise en charge médicale des individus sans-domicile peuvent pousser les infirmiers à agir de manière contraire à leur éthique du care. Toutefois, des dispositifs spécialisés dans la prise en charge médicale des personnes sansabri, apportent des possibilités de surmonter leurs difficultés d'intercompréhension. Bien qu'il ne s'agisse pas de solutions miracles garantissant une prise en charge optimale des patients, ces espaces-temps intermédiaires permettent aux infirmiers d'appliquer leur éthique du care. En effet, il ne leur suffit pas de défendre ces valeurs du soin pour pouvoir les mettre en pratique, l'espace-temps dans lequel ils évoluent doit leur apporter les conditions nécessaires, notamment en termes de temps à consacrer aux patients, pour que l'éthique du care puisse être effective. Pour que le care soit mis en pratique de manière sereine, la sécurité ontologique du soignant et du soigné doit être préservée au mieux.

En conclusion, on observe que c'est la situation particulière d'interaction qui rend vulnérables infirmiers et personnes de la rue, car elle fait s'entrechoquer leurs valeurs et représentations. Comme le rappelle Patrice Bourdelais, «la vulnérabilité des personnes est rarement due à une caractéristique intrinsèque, même si elle peut exister: Achille n'était vulnérable que si une agression extérieure atteignait son talon» (Bourdelais 2006, p. 6). Il en est de même ici, d'une part, les patients se sentent malmenés, rejetés, stigmatisés lorsqu'ils ont l'impression que les soignants ne leur accordent pas l'at- 
tention dont ils ont besoin; et d'autre part, la prise en charge médicale de ces patients engendre un sentiment d'inutilité chez les infirmiers, qui se sentent démunis face à des prises en charge lourdes, auxquelles ils n'ont que peu de réponses à apporter. La création d'une relation soignant-soigné basée sur la confiance et la reconnaissance réciproque est alors compromise, alors même qu'elle représente la condition sine qua non à la mise en pratique du care puisqu'elle offre à chacun une protection de sa sécurité ontologique. Les infirmiers se retrouvent en contradiction avec leur éthique du care. Ils expliquent en effet que c'est grâce à cette relation que le soin peut s'opérer dans des conditions idéales. Elle leur permet de mieux appréhender l'état de santé et les différents symptômes du patient, qui se livrera plus facilement s'il a confiance en l'infirmier. La relation est également nécessaire pour faire adhérer aux soins les patients via l'explication de la pathologie et des différents soins utilisés pour la traiter, d'autant plus lorsqu'il s'agit de patients souvent réfractaires aux soins et qui éprouvent des difficultés à appréhender leur pathologie comme les patients sans-domicile. Enfin et surtout, la relation soignant-soigné permet d'accorder aux deux parties la reconnaissance dont ils manquent. Si le care était rasséréné, moins malmené, alors les infirmiers s'estimeraient valorisés dans leur travail et fidèles à leurs valeurs de soignants et les personnes sans-abri se sentiraient reconnues en tant qu'individus qui ont de la valeur et dont il faut prendre soin.

\section{Bibliographie}

Bourdelais P. (2006), Qu'est-ce que la vulnérabilité?, Annales de démographie historique, 110 (2), p. 5-9.

Canguilhem G. (1972), Le normal et le pathologique, Paris, Presses universitaires de France.

Fainzang S. (2006), La relation médecins-malades: information et mensonge, Paris, Presses universitaires de France.

Freidson E. (1984), La profession médicale, Paris, Payot.

Freund J. (1983), Sociologie du conflit, Paris, Presses universitaires de France.
Giddens A. (2012), La constitution de la société: éléments de la théorie de la structuration, Paris, Presses Universitaires de France.

Girola C. M. (2011), Vivre sans abri: de la mémoire des lieux à l'affirmation de soi, Paris, Éditions Rue d'Ulm.

Goffman E. (1947), Les rites d'interaction, Paris, Les Éditions de Minuit.

Herzlich C. (1970), Médecine, maladie et société, Paris, Mouton.

Honneth A. (2000), La lutte pour la reconnaissance, Paris, Éditions du Cerf.

Klinger M. (2003), Services publics et vulnérabilité: L'accueil des incertitudes, Annales de la recherche urbaine (94), p. 49-52.

Le Breton D. (1992), La sociologie du corps, Paris, Presses universitaires de France.

Pichon P. (2010), Vivre dans la rue: sociologie des sans domicile fixe, Saint-Étienne, Publications de l'Université de Saint-Étienne.

Pulcini E. (2012), Donner le care, Revue $d u$ MAUSS (1), p. 49-66.

Simmel G. (1999), Sociologie: étude sur les formes de la socialisation, Paris, Presses universitaires de France.

Tronto J. C. (2008), Du care, Revue du MAUSS (2), p. 243-265.

Véga A. (1997), Les infirmières hospitalières françaises: l'ambiguité et la prégnance des représentations professionnelles, Sciences sociales et santé 15 (3), p. 103-132.

\section{Notes}

1. Anthony Giddens explique que «la vie quotidienne suppose une sécurité ontologique qui est l'expression de l'autonomie de contrôle corporel dans des routines prévisibles» (Giddens 2012, p. 99). 